\title{
CONNECTED PLANE SETS WHICH CONTAIN NO NONDEGENERATE CONNECTED SIMPLE GRAPH
}

\author{
B. D. GARRETT
}

(Communicated by James E. West)

\begin{abstract}
Open questions are answered by showing that, if for each vertical line $L$ in the plane, $1<k_{L} \leq c$ is a cardinal number, there is a connected subset $H$ of the plane such that, if $L$ is a vertical line, then $L \cap H$ has cardinality $k_{L}$ and, if $g$ is a nondegenerate connected subset of $H$, then $g$ contains two points of some vertical line.
\end{abstract}

\section{INTRODUCTION}

The arguments herein answer questions which have remained open for a number of years. Often stated in other terminology, those questions are of the following form. If $k$ is a cardinal number greater than 1, does there exist a connected subset $G$ of the plane such that, each vertical line contains $k$ points of $G$ and such that, if $g$ is a connected subset of $G$, with $x$-projection $R$, then $g$ contains two points of some vertical line. Though such questions were well known earlier, special cases began to appear in print as early as 1972 [1].

F. B. Jones [4] answered a question from [1] when he presented a solution to the case where $k=2$, (at most,) in 1974. In 1983, T. Tanaka [7] also gave a solution. In 1975, Jones [5] answered a modified version due to P. Erdös, solving the case where $k$ is 2 . The case where $k=\aleph_{0}$ was solved by Ceder in [2] along with the case where $k$ is $c$. In that same paper, Ceder introduced the added restriction on the set $G$ that it be "strongly connected." His solution for $k=c$ is strongly connected but none of the other solutions mentioned above is strongly connected. Ceder left, as an open question, the possibility of there being a strongly connected example for the case for $k=\aleph_{0}$. Such an example is included in the result of the present paper. Clearly, neither Ceder nor Tanaka was aware of Jones' example [5]. Probably the best known such problem is from Erdös and appears in [6] as Problem G.1: Is there a connected set in the plane which meets each vertical line in precisely two points such that every nondegenerate connected subset meets some vertical line in two points?

Received by the editors July 25, 1988 and, in revised form, April 26, 1989.

1980 Mathematics Subject Classification (1985 Revision). Primary 54C08; Secondary 54C65, $54 \mathrm{C} 60$.

Key words and phrases. Simple graph, selection, connectivity maps. 
The arguments below will prove that, if for each vertical line $L$ in the plane, $1<k_{L} \leq c$ is a cardinal number, there is a strongly connected subset $S$ of the plane such that, for each vertical line $L$, the set $S \cap L$ has cardinality $k_{L}$ and, if $g$ is a nondegenerate connected subset of $S$, some vertical line contains two points of $g$. Because strongly connected sets must be connected, this includes a solution to the problem of Erdös stated above.

\section{PRELIMINARIES}

A subset of the plane is called a graph. The $x$-projection of a graph $f$ is denoted by $(f)_{X}$ and the $y$-projection is $(f)_{Y}$. Whenever $A$ is a point or subset of $(f)_{X}$, the set $f \mid A$, the restriction of $f$ to $A$, is the set of all points of $f$ with abscissa in $A$. Then, for any graph $f$ and any $A$ in $(f)_{X}$, we have $f(A)=(f \mid A)_{Y}$.

If $f$ is a graph such that, for each point $r$ in $(f)_{X}$, the set $f(r)$ has at most $k$ members, the graph $f$ is called a $k$-graph; if each $f(r)$ has exactly $k$-members, $f$ is an exact $k$-graph. That $f$ is a simple graph means that $f$ is a 1-graph. When $f$ is a simple graph, $f(+)$ is the set $\left\{(r, s): r\right.$ is in $(f)_{X}$, $s>f(r)\}$ and $f(-)=\left\{(r, s): r\right.$ is in $\left.(f)_{X}, s<f(r)\right\}$.

Certain notations are reserved throughout. The capital letter $K$ is the Cantor middle thirds set of the closed unit interval $[0,1]=I$. By the "end points" of a Cantor set $L$, we mean the set of all end points of components of $R-L$, where $R$ is the real line. The capital letter $E$ always is the set of end points of $K$. The capital letters $F, G$, and $H$ are specific graphs defined by constructions and $A=I \times \frac{1}{2}$. Various other graphs and sets used in those constructions will be given notations that will be used to refer to them later.

The notation $(a, b)$ may refer to either the point in $R^{2}$ with coordinates $a$ and $b$ or it may be an open connected subset of $R$, depending on the conditions of its use. Of course, the closure of the open interval $(a, b)$ is $[a, b]=\operatorname{cl}[(a, b)]$ and $\operatorname{int}[a, b]=(a, b)$. When used in notations such as $M \times r, M-r$, etc., where $r$ is obviously the name of some number or point, then $r$ refers to the set whose only element is the number or point $r$. A continuum is a closed, connected, and bounded subset of the plane. The real line has $c$-many points.

Definition 1. The connected graph $f$ is strongly connected means that, if $(a, b)$ is an open interval in $(f)_{X}$, then $f \mid(a, b)$ is connected.

Definition 2. The point $(r, s)$ of the graph $f$ is a limit point of $f$ from the left if $(-\infty, r) \times R$ contains a subset of $f$ having $(r, s)$ as a limit point; it is a limit point of $f$ from the right if $(r, \infty) \times R$ contains a subset of $f$ having $(r, s)$ as a limit point.

The proofs of Properties 1 and 3 are standard. Property 2 is proved in Theorem 2 of [3]. 
Property 1. If the simple graph $f$ is connected, $r$ and $s$ are in $(f)_{X}$, and $r<s$, then $r$ is a limit point of $f$ from the right and $s$ is a limit point of $f$ from the left.

Property 2. The simple graph $f$ is connected if and only if $f$ intersects each continuum which intersects both $f(+)$ and $f(-)$.

Property 3. If the continuum $M \subset I^{2}$ contains the point $P$ of $(K-E) \times I$ and $(M)_{X}$ is nondegenerate, then $P$ lies in a subcontinuum $N$ of $M$ with $(N)_{X}$ nondegenerate and containing $c$-many points of $K$. Moreover, if $P$ lies above $I \times 0$ and $M$ contains no point of $E \times 0$, then $N$ may be chosen above $I \times 0$.

The development below procedes through the construction of three successively more complicated graphs, $F, G$, and $H$. Through the constructions we will show that:

(a) the graph $F$ exists, has $(F)_{X}=I$, is strongly connected, contains at most two points of any vertical line, and contains no connected simple graph with $x$-projection $I$;

(b) the graph $G$ exists, has $(G)_{X}=I$, is strongly connected, contains at most two points of any vertical line, and contains no connected simple graph with nondegenerate $x$-projection;

(c) if for each $r$ in $I$, there is a cardinal number, $1<k_{r} \leq c$, then the graph $H$ exists, has $(H)_{X}=I$, for $r$ in $I, H \cap(r \times R)$ has exactly $k_{r}$ points, and no nondegenerate connected subset of $H$ is a simple graph.

With (a), (b), and (c), we have improved results from $[2,4,5,7]$.

\section{THE GRAPH $F$}

Denote by $B$ the set to which the continuum $M \subset I^{2}$ belongs only if there is a subset $N_{M}$ of $K-E$ with $c$-many elements such that, if $r$ is in $N_{M}$, then $r \times\left(\left(0, \frac{1}{2}\right) \cup\left(\frac{1}{2}, 1\right]\right)$ intersects $M$. There are exactly $c$-many continua in $B$ and there is a well ordering $\beta$ of the continua in $B$ so that no term of $\beta$ is preceded by $c$-many terms of $\beta$. Construct a simple graph $b$, with $(b)_{X}=K-E$ as follows. By induction on the well ordering $\beta$, for each continuum $M_{u}$ of $\beta$, select a point $\left(r_{u}, b\left(r_{u}\right)\right)$ of $M_{u}$ such that $r_{u}$ is in $N_{M_{u}}$ and $0 \neq b\left(r_{u}\right) \neq \frac{1}{2}$ and, if $M_{V}$ is some other continuum of $\beta, r_{V} \neq r_{u}$. If $r$ is in $K-E$ but has not been selected as the abscissa for a point $\left(r_{u}, b\left(r_{u}\right)\right)$ in a continuum $M_{u}$ of $\beta$, then take $(r, b(r))=(r, 1)$.

With $F=b \cup\left(E \times\left\{0, \frac{1}{2}\right\}\right) \cup([I-K] \times 0)$ each of the following statements is true.

(1) $(F)_{X}=I$.

(2) For each $r$ in $I-K$, the set $F \mid r$ contains only one point and it has ordinate 0 .

(3) For each $r$ in $K-E$, the set $F \mid r$ contains only one point and it has ordinate in $(0,1]$, which is different from 0 or $\frac{1}{2}$.

(4) For each $r$ in $E$, the set $F \mid r$ contains exactly two members, one with ordinate 0 and the other with ordinate $\frac{1}{2}$. 
(5) $F \cap A=F \cap\left(I \times \frac{1}{2}\right)=F \cap\left(K \times \frac{1}{2}\right)=E \times \frac{1}{2}$ is a countable dense subset of $K \times \frac{1}{2}$.

(6) $F \cap(K \times 0)=E \times 0$ is a countable dense subset of $K \times 0$.

(7) If $U \subset I^{2}$ is open and contains a point of $F \mid K$, then $U$ contains a point of $F \mid(K-E)$.

Theorem 1. Suppose $M \subset I^{2}$ is a continuum such that $(M)_{X}$ is nondegenerate, $M$ contains no point of $E \times 0$, and $M$ intersects $(K-E) \times I$. Then $M$ contains a point of $F$.

Proof. Clearly, $M_{X} \cap(K-E)$ is at most nowhere dense in $K$. Then, using Property 3 , since $M$ contains a point $(r, y), y>0$, of $(K-E) \times I$, it contains a subcontinuum $N$, containing $(r, y)$, with $(N)_{X}$ nondegenerate and $(N)_{Y} \subset$ $(0,1]$. If $K \cap\left(N-\left[K \times \frac{1}{2}\right]\right)_{X}$ has less than $c$-many points, then $N \cap\left(K \times \frac{1}{2}\right)$ is of the second category in $K \times \frac{1}{2}$ and contains a point of $\left(E \times \frac{1}{2}\right) \subset F$. In that case, the set $M$ is closed; so $M$ has a point in $\left(E \times \frac{1}{2}\right) \subset F$. If $\left(N-\left[K \times \frac{1}{2}\right]\right)_{X}$ has $c$-many points, $M$ contains a point of $F$ via the construction of $F$.

Theorem 2. The graph $F$ is strongly connected.

Proof. Assume $C \subset I$ is a connected open set for which $F \mid C$ is not connected. There is a continuum $M \subset \operatorname{cl}(C) \times I$ containing no point of $F \mid C$ and separating $F \mid C$ in $\operatorname{cl}(C) \times I$. It is obvious that $(M)_{X}$ is nondegenerate. Since $M$ contains no point of $F$, it contains no point of $E \times 0$. If $(M)_{X}$ is a subset of $\operatorname{cl}(V)$, where $V$ is a component of $C-(C \cap K)$, then $S=[\operatorname{cl}(F \mid U)] \cup([C-\operatorname{cl}(V)] \times I)$ is connected and contains no point of $M$. Each point of $F \mid(\operatorname{cl}(V)-V)$ is a limit point of $S$ and is not in $M$. In this case, $M$ cannot separate $F$ in $\operatorname{cl}(C) \times I$. We are left with the conclusion that $M$ intersects $(K-E) \times I$. By Theorem $1, M$ contains a point of $F$. Thus $F \mid C$ is connected whenever $C$ is a connected open set of $I$.

Theorem 3. The graph $F$ contains no connected simple graph which has $x$ projection $I$.

Proof. Assume $g \subset F$ is a connected simple graph with $(g)_{X}=I$. If $\left(e, \frac{1}{2}\right)$ is any point of $A \cap F$, either $F \mid(e, 1]$ or $F \mid[0, e)$ has $\left(e, \frac{1}{2}\right)$ as a limit point but not both. By Property 1 , if $e$ is a point of $E$, the point $\left(e, \frac{1}{2}\right)$ cannot be a point of $g$. Then $(e, 0)=(e, g(e))$ is a point of $g$ below $A$. There is an $r$ in $K-E$ such that the only point of $F \mid r$ is above the continuum $A$. Clearly, $(r, g(r))$ is above the continuum $A$ and $(e, g(e))$ is below the continuum $A$. Property 2 requires that $A$ contain a point of $g$, which is impossible.

\section{THE GRAPH $G$}

For any interval $[a, b] \subset I$, the homeomorphism,

$$
T_{(a, b)}: I^{2} \rightarrow[a, b] \times[0, b-a]
$$

is defined by

$$
T_{(a, b)}(x, y)=(a+x(b-a), y(b-a)) \text {. }
$$


With $K_{0}=K \mid(0,1)$, if $p$ is a positive integer, denote the nondegenerate components of the complement, in $I$, of the set $K_{p-1}$ by $\left(a_{p q}, b_{p q}\right), q=$ $1,2,3, \cdots$, where

$$
K_{p}=K_{p-1} \bigcup_{q=1}^{\infty}\left(\cup K_{p q}\right)
$$

and, for each $q=1,2,3, \cdots$,

$$
K_{p q}=T_{\left(a_{p q}, b_{p q}\right)}\left(K_{0}\right) .
$$

Using $f_{0}=F \mid(0,1)$, for each positive integer $p$, the graph $f_{p}$ is defined by

$$
f_{p}=\left(f_{p-1}-\bigcup_{q=1}^{\infty}\left[\left(a_{p q}, b_{p q}\right) \times 0\right]\right) \bigcup_{q=1}^{\infty}\left(\cup f_{p q}\right)
$$

where, for each $q=1,2,3, \cdots$,

$$
f_{p q}=T_{\left(a_{p q}, b_{p q}\right)}\left(f_{0}\right) .
$$

The graph $G$ is $S \cup\left(F-f_{0}\right)$, where $S$ is the set to which the point $P$ belongs only if there is a positive integer $n$ such that, if $m>n$, the graph $f_{m}$ contains $P$.

For notational convenience, if $p=0$, we will say $f_{p q}=f_{0}, a_{p q}=0$, and $b_{p q}=1$. Then, if $p$ is a nonnegative integer and $q$ is a positive integer, an "integer pair $(p, q)$ " uniquely determines, via the construction above, the sets $f_{p q}, k_{p q}$, etc. For any such integer pair $(p, q), E_{p q}$ is the set of end points of $\operatorname{cl}\left(K_{p q}\right)$ in $\left(a_{p q}, b_{p q}\right)$ and $\alpha_{p q}=T_{\left(a_{p q}, b_{p q}\right)}(A \mid(0,1))$.

It is clear that each of the following statements is true.

(1) $(G)_{X}=I$.

(2) If $r$ is in $I-\left(K \cup\left[\bigcup_{m=1}^{\infty} K_{n}\right]\right)$, the set $G \mid r$ has only one point and it has ordinate 0 .

(3) If $r$ is in $K_{p q}-E_{p q}$, the set $G \mid r$ contains only one point and it has ordinate, other than 0 or $\frac{1}{2}\left(b_{p q}-a_{p q}\right)$, in $\left(0, b_{p q}-a_{p q}\right)$.

(4) For $r$ in any $E_{p q}$, the set $G \mid r$ has exactly two members, one with ordinate 0 and the other with ordinate $\frac{1}{2}\left(b_{p q}-a_{p q}\right)$.

(5) $G \cap \alpha_{p q}=G \cap\left[\left(a_{p q}, b_{p q}\right) \times \frac{1}{2}\left(b_{p q}-a_{p q}\right)\right]=G \cap\left[K_{p q} \times \frac{1}{2}\left(b_{p q}-a_{p q}\right)\right]=$ $E_{p q} \times \frac{1}{2}\left(b_{p q}-a_{p q}\right)$ is a countable dense subset of $K_{p q} \times \frac{1}{2}\left(b_{p q}-a_{p q}\right)$.

(6) $G \cap\left(K_{p q} \times 0\right) \stackrel{p q}{=}\left(E_{p q} \times 0\right)$ is a countable dense subset of $K_{p q} \times 0$.

(7) If $V \subset I^{2}$ is open and contains a point of $G \mid K_{p q}$, then $V$ contains a point of $G \mid\left(K_{p q}-E_{p q}\right)$.

(8) If $V \subset I$ is open, there is some $\left(a_{p q}, b_{p q}\right) \subset V$.

(9) There is a dense subset $S$ of $K_{p q}$ such that, if $r$ is in $S$, the set $(G \mid r)_{y}$ contains a single point in $\left(\frac{1}{2}\left(b_{p q}-a_{p q}\right), b_{p q}-a_{p q}\right]$. 
Theorem 4. The graph $G$ is strongly connected.

Proof. Assume $G$ is not strongly connected. There is an open connected $C \subset I$ and a continuum $M \subset \operatorname{cl}(C) \times I$ such that $M$ separates $G \mid C$ in $[\operatorname{cl}(C) \times I]$ but contains no point of $G \mid C$. It is obvious that $(M)_{X}$ is nondegenerate.

Suppose $(p, q)$ is an integer pair and that $M$ intersects $\left(K_{p q}-E_{p q}\right) \times$ $\left(0, b_{p q}-a_{p q}\right]$. There is a subcontinuum $N$ of $M$, with $(N)_{X} \subset C \cap\left(a_{p q}, b_{p q}\right]$, such that each end point of $(N)_{X}$ is in $\left(K_{p q}-E_{p q}\right)$ and $(N)_{Y} \subset\left(0, b_{p q}-\right.$ $\left.a_{p q}\right)$. Therefore, we have a continuum $N^{*}$ in $I \times(0,1]$, defined by $N^{*}=$ $T_{\left(a_{p q}, b_{p q}\right)}(N)$. Theorem 1 tells us that the continuum $N^{*}$ contains a point of $F \mid(0,1)$. That means that $N$ must contain a point of $G$. This is a contradiction, proving that $(*) M$ cannot contain a point of $\left(K_{p q}-E_{p q}\right) \times\left(0, b_{p q}-a_{p q}\right]$, for any integer pair $(p, q)$.

Either $M$ contains a point of $C \times 0$ or it does not. Suppose $(r, 0)$ is a point of $M$ in $C \times 0$. This gives that $(r, 0)$ is not in $G$, implying that $r$ is in some $\left(K_{p q}-E_{p q}\right)$. The nondegenerate set $(M)_{X}$ has some other point $s$ in $K_{p q}-E_{p q}$. Between the two points, $r$ and $s$, of $\left(K_{p q}-E_{p q}\right)$, there must be a point $e$ of $E_{p q}$. Since $e$ is in $(M)_{X}$ and $(e, 0)$ is not in $M$, there is a point $(e, y)$ of $M$ with $y>0$. Clearly, there is a point of $M$ in $\left(K_{p q}-E_{p q}\right) \times\left(0, b_{p q}-a_{p q}\right]$, contradicting $(*)$. Therefore, $M$ does not contain a point of the connected set $(C \times 0)$.

In the case where $M$ does not intersect $C \times 0$, there are two components, $U_{1}$ and $U_{2}$, of $[\operatorname{cl}(C) \times I]-M$ such that, $(C \times 0) \subset U_{1}$ and $U_{2}$ contains a point $(x, y)$ of $G$, with $x$ in $C$ and $y>0$. There is an $\varepsilon>0$ such that, if $r$ is in $[x-\varepsilon, x+\varepsilon]$, then $(r, y)$ is in $U_{2}$. Clearly, the continuum $M$ contains a point of every $r \times(0, y)$, where $r$ is in $[x-\varepsilon, x+\varepsilon]$. The point $(x, y)$ is in some $f_{p q}$ so that $x$ is in $K_{p q}$. Since $\left(K_{p q}-E_{p q}\right)$ is dense in $K_{p q}$, there is a point $k$ of $\left[K_{p q}-E_{p q}\right]$ in $[x-\varepsilon, x+\varepsilon]$ and a point of $M$ in $k \times(0, y)$. Thus, $M$ contains a point of $\left(K_{p q}-E_{p q}\right) \times\left(0, b_{p q}-a_{p q}\right]$, contradicting $(*)$. There is no further case, so our original assumption that $G$ is not strongly connected, is false.

Theorem 5. The graph $G$ contains no connected simple graph with nondegenerate $x$-projection.

Proof. Assume $g \subset G$ is a connected simple graph with $(g)_{X}$ nondegenerate. There is an integer pair $(p, q)$ such that $\left(f_{p q}\right)_{X} \subset(g)_{X}$. Consider the horizontal open interval $\alpha_{p q}$ and $\alpha_{p q} \cap G=E_{p q} \times \frac{1}{2}\left(b_{p q}-a_{p q}\right)$. No point $(r, y)$ of $\alpha_{p q}$ is a limit point of both $f_{p q} \mid\left(a_{p q}, r\right)$ and $f \mid\left(r, b_{p q}\right)$. If $(m, n)$ is an integer pair such that $m \neq p$ or $n \neq q$, either $\left(f_{m n}\right)_{X}$ has no limit point in $\left(a_{p q}, b_{p q}\right)$ or $\operatorname{cl}\left(f_{m n}\right)$ lies entirely below $\alpha_{p q}$. Thus, no point of $G \cap \alpha_{p q}=E_{p q} \times \frac{1}{2}\left(b_{p q}-a_{p q}\right)$ is a point of $g$. Clearly, each point of $\left(E_{p q} \times 0\right)$ is a point of $g$. For $r$ in $K_{p q}-E_{p q}$, the set $G \mid r$ has only one member, so it is $(r, g(r))$. There is then an $r$, in $K_{p q}-E_{p q}$, for which $(r, g(r))$ is above $\alpha_{p q}$. Also, there is some $e$ 
in $E_{p q}$, with $e<r$. Now $(e, g(e))$ is below $\alpha_{p q} \mid[e, r]$. By Property 2 , the continuum $\alpha_{p q} \mid[e, r]$ contains a point of $G$. This is a contradiction.

\section{THE GRAPH $H$}

The set $S$, to which $r$ belongs only if $r$ is in $(0,1)$ but belongs to no $K_{p}$ is of the second category in every open set of $I$. If $M$ is a continuum with $(M)_{X} \subset I$ nondegenerate, $(M)_{X} \cap S$ is of the second category in $I$ and has $c$-many points. There are $c$-many continua with nondegenerate $x$-projection in $I$ and with $y$-projection in $[-1,0)$. Denote by $\beta$ a well ordering of those continua so that no term of $\beta$ is preceded by $c$-many terms of $\beta$. Define a simple graph $t$ with $(t)_{X}=S$ as follows. Using induction on the well-ordering $\beta$, for each continuum $M_{u}$ in $\beta$, select a point $\left(r_{u}, t\left(r_{u}\right)\right)$ of $M$ so that, $r_{u}$ is in $S$ and, if $M_{v}$ is any other term of $\beta, r_{v} \neq r_{u}$. If there is a point $r$ of $S$ not assigned to a point $\left(r_{u}, t\left(r_{u}\right)\right)$ by the induction, then $(r,-1)=(r, t(r))$.

Suppose, for each number $r$ in $I, 1<k_{r} \leq c$ is a cardinal number. Define the graph $H$ as follows. If $r$ is in $(t)_{X}$, then $(H \mid r)_{Y}$ is a totally disconnected set with cardinality $k_{r}$, containing $(G \mid r)_{Y}$ and $t(r)$ but otherwise lying in $\left[-\frac{1}{2}\right.$, $-1]$. If $r$ is in some $\left(f_{p q}\right)_{X}$, then $(H \mid r)_{Y}$ is a totally disconnected set with cardinality $k_{r}$, containing $(G \mid r)_{Y}$, and any other point of it is in $\left(\frac{1}{2}\left(b_{p q}-a_{p q}\right)\right.$, $b_{p q}-a_{p q}$ ]. If $r$ is either 0 or 1 , then $(H \mid r)_{Y}$ is a totally disconnected set, with cardinality $k_{r}$, containing $(G \mid r)_{Y}$, and any other of its points are in $\left(\frac{1}{2}, 1\right]$.

The graph $H$ has the following properties.

(1) $(H)_{X}=I$.

(2) If $r$ is in $I-\left(K \cup\left[\bigcup_{m=1}^{\infty} K_{n}\right]\right)$, the set $H \mid r$ contains $(r, 0)$ and lies otherwise below the $x$-axis.

(3) If $r$ is in $K_{p q}-E_{p q}$, the set $(H \mid r)_{Y}$ lies in $\left(0, b_{p q}-a_{p q}\right]$ but does not contain $\frac{1}{2}\left(b_{p q}-a_{p q}\right)$ or 0 ; if $G \mid r$ lies above $\alpha_{p q}$, then $H \mid r$ does also.

(4) For $r$ in $E_{p q}$, the set $(H \mid r)_{Y}$ contains 0 and $\frac{1}{2}\left(b_{p q}-a_{p q}\right)$ and lies otherwise in $\left(\frac{1}{2}\left(b_{p q}-a_{p q}\right), b_{p q}-a_{p q}\right]$.

(5) $H \cap \alpha_{p q}=H \cap\left[\left(a_{p q}, b_{p q}\right) \times \frac{1}{2}\left(b_{p q}-a_{p q}\right)\right]=H \cap\left[K_{p q} \times \frac{1}{2}\left(b_{p q}-a_{p q}\right)\right]=$ $E_{p q} \times \frac{1}{2}\left(b_{p q}-a_{p q}\right)$ is a countable dense subset of $K_{p q} \times \frac{1}{2}\left(b_{p q}-a_{p q}\right)$.

(6) $H \cap\left(K_{p q} \times 0\right)=E_{p q} \times 0$ is a countable dense subset of $K_{p q} \times 0$.

(7) If $V \subset I^{2}$ is open and contains a point of $H \mid K_{p q}$, then $V$ contains a point of $H \mid\left(K_{p q}-E_{p q}\right)$.

(8) If $V \subset I$ is open, there is some $\left(a_{p q}, b_{p q}\right) \subset V$.

(9) There is a dense subset $S$ of $K_{p q}-E_{p q}$ such that, if $r$ is in $S$, then $H \mid r$ is above $\alpha_{p q}$.

(10) $\operatorname{cl}\left(\left[G \cap I^{2}\right] \mid C\right)=\operatorname{cl}\left(\left[H \cap I^{2}\right] \mid C\right)$, if $C \subset I$ is open.

(11) For each $r$ in $I$, the cardinality of $H \mid r$ is $k_{r}$.

Theorem 6. The graph $H$ is strongly connected. 
Proof. Suppose $C \subset I$ is open and connected. By Theorem 4, $G \mid C$ is connected. From the construction of $H$, we have $G\left|C \subset\left(H \cap I^{2}\right)\right| C$ and $\left[\left(H \cap I^{2}\right) \mid\right.$ $C] \subset[\operatorname{cl}(G \mid C)]$. Thus $\left(H \cap I^{2}\right) \mid C$ is connected.

Now assume $M \subset \operatorname{cl}(C) \times[-1,1]$ separates $H \mid C$ in $\operatorname{cl}(C) \times[-1,1]$ but contains no point of $H \mid C$. There is a component $U_{1}$ of $(\operatorname{cl}(C) \times[-1,1])-M$ containing $\left(H \cap I^{2}\right) \mid C$ and a component $U_{2}$ of $(\mathrm{cl}(C) \times[-1,1])-M$ containing a point $(r, s)$ of $H$ such that $r$ is in $C$ and $s<0$. Then $M$ must contain a point of $r \times(s, 0)$, because both $(r, s)$ and $(r, 0)$ are in $H$, the point $(r, s)$ is in $U_{2}$, and the point $(r, 0)$ is in $U_{1}$. Since a subcontinuum of $r \times(s, 0)$ cannot separate $H$ in $\operatorname{cl}(C) \times[-1,1], M$ contains a point $(x, y)$, where $x \neq r$. Clearly $M$ has a subcontinuum $N$ containing $(r, s)$ with $(N)_{X}$ nondegenerate and $(N)_{Y} \subset[-1,0)$. By the construction of $H$, the continuum $N$ contains a point of $H$ and we have reached a contradiction. Thus $H$ is strongly connected.

Theorem 7. The graph $H$ contains no connected simple graph with nondegenerate $x$-projection.

Proof. This is an obvious variation of the argument used to prove Theorem 5.

Theorem 8. If for each $r$ in $R, 1<k_{r} \leq c$ is a cardinal number, there is a strongly connected graph $f$ such that, if $r$ is in $R$, the set $f \mid r$ has cardinality $k_{r}$ and, if $g$ is any nondegenerate connected subset of $f$, then $g$ is not a simple graph.

Proof. There is a homeomorphism $h:(0,1) \rightarrow R$. Such a graph, as $f$, results from using Theorem 7 and $h$.

Note. While at the symposium referenced in [4], the author participated in an informal discussion. Its other three participants were F. B. Jones, Stephen Curry, and Carl Seaquist. Years later, in January 1988, quite by accident, the author found several pages of notes he had taken during that discussion. Within those notes, through a sequence of drawings and accompanying descriptions, he recognized the basic line of argument of the present paper. Each of the above named persons denied any responsibility for the arguments here and refused to participate as a co-author. Thus, the author accepts all responsibility for the content of this paper but wonders if he is the sole author by default.

\section{REFERENCES}

1. A. M. Bruckner and J. Ceder, On jumping functions by connected sets, Czech. Math. J. 22 (1972), 435-448.

2. J. Ceder, On Darboux selections, Fund. Math. 91 (1976), 85-91.

3. B. D. Garrett, D. Nelms, and K. R. Kellum, Characterizations of connected real functions, Jahr. Deutsch. Math. 73 (1971), 131-137.

4. F. B. Jones, Connected functions in the connected union of functions, presented at the Symposium on Pure and Applied Mathematics in Memory of Pasquale Porcelli, University of Houston, November, 1974. 
5. __ Connected simple graphs and a selection problem, Czech. Math. J. 25 (1975), 300-301.

6. M. E. Rudin, Lectures on set theoretic topology, Regional Conference Series in Mathematics, no. 23, Amer. Math. Soc., Providence, R.I., 1974.

7. T. Tanaka, A question of Ceder concerning connected selections, Questions Answers Gen. Top. 1 (1983), 144-148.

Department of Physics, Mathematics, and Computer Science, Tennessee State University, NAShVille, TenNessee 37203

Current address: 1001 Alice, Denton, Texas 76201 